\title{
bZIP17 regulates heat stress tolerance at reproductive stage in Arabidopsis
}

\author{
Juan Gao' ${ }^{1}$, Mei-Jing Wang ${ }^{1}$, Jing-Jing Wang ${ }^{1}$, Hai-Ping Lu ${ }^{1}$, \\ Jian-Xiang Liu ${ }^{1 凶}$ \\ ${ }^{1}$ State Key Laboratory of Plant Physiology and Biochemistry, College of Life Sciences, Zhejiang University, \\ Hangzhou 310027, China
}

Received: 26 September 2021 / Accepted: 30 October 2021 / Published online: 25 November 2021

\begin{abstract}
High temperature elicits a well-conserved response called the unfolded protein response (UPR) to bring protein homeostasis in the endoplasmic reticulum (ER). Two key UPR regulators bZIP28 and bZIP60 have been shown to be essential for maintaining fertility under heat stress conditions in Arabidopsis, however, the function of transcriptional activator bZIP17, a paralog of bZIP28, in heat stress response at reproductive stage is not reported. Here we found that bzip17 mutant plants were sensitive to heat stress in terms of silique length and fertility comparing to that of wildtype (WT) Arabidopsis plants, and transcriptomic analysis showed that 1380 genes were specifically up-regulated and 493 genes were specifically down-regulated by heat stress in the flowers of WT plants comparing to that in bzip17 mutant plants. These bZIP17-dependent up-regulated genes were enriched in responses to abiotic stresses such as water deprivation and salt stress. Further chromatin immuno-precipitation coupled with high-throughput sequencing (ChIP-Seq) uncovered 1645 genes that were direct targets of bZIP17 in MYC-bZIP17 expressing seedlings subjected to heat stress. Among these 1645 genes, ERSE-II ciselement was enriched in the binding peaks of their promoters, and the up-regulation of 113 genes by heat stress in flowers was dependent on bZIP17. Our results revealed direct targets of bZIP17 in flowers during heat stress responses and demonstrated the important role of bZIP17 in maintaining fertility upon heat stress in plants.
\end{abstract}

Keywords Arabidopsis, bZIP17, Flower, Fertility, Heat Stress, UPR

\section{INTRODUCTION}

Global warming has great impact on plant growth and food production, as a yield loss of $6-7 \%$ per $1{ }^{\circ} \mathrm{C}$ increase in seasonal mean weather associated with extreme heat stress predicted (Lesk et al. 2016). Understanding on how plants perceive and respond to high temperature signals, especially at reproductive stage, is vital for molecular breeding of temperature stress resilient crops (Zhang et al. 2019).

$\bowtie$ Correspondence: jianxiangliu@zju.edu.cn (J.-X. Liu)
A primary effect of heat stress on cellular function in plants is denaturing proteins and causing protein aggregations both in the cytosol and endoplasmic reticulum (ER) (Ding et al. 2020). Therefore, intracellular protein homeostasis is important to maintain the protein conformation, status, level and activity during heat stress (Sun et al. 2021). Indeed, a major thermotolerance quantitative trait loci (QTL) from African rice (Oryza glaberrima), Thermotolerance 1 (OgTT1), was identified, and it encodes an $\alpha 2$ subunit of $26 \mathrm{~S}$ proteasome responsible for eliminating cytotoxic denatured proteins (Li et al. 2015). A number of heat shock proteins (HSPs), such as HSP100s, HSP90s, HSP70s, 
HSP60s, HSP40s and sHSPs with different molecular weight, have been characterized with the major functions of these proteins as molecular chaperons in cytoplasm during heat stress responses (Jacob et al. 2017; Nover and Scharf 1997). In addition, heat stress transcription factors (HSFs) play a crucial role in heat stress responses through regulating the expression of HSPs, providing heat stress tolerance in plants (Guo et al. 2016).

Disturbance of protein folding homeostasis in ER under heat stress conditions elicits a well-conserved unfolded protein response (UPR), in which several membrane-associated transcription factors sense and transduce the stress signals to nucleus to regulate downstream genes, bringing back the protein homeostasis (Liu and Howell 2016). Upon the accumulation of misfolded proteins in the ER, the Arabidopsis ER membrane-associated transcription factor AtbZIP28 is translocated from ER to Golgi where it is subjected to intramembrane proteolysis by Golgi-localized proteases (Che et al. 2010; Gao et al. 2008; Liu et al. 2007a). The activated AtbZIP28 enters nucleus and regulates downstream genes to enhance protein folding capacity and accelerate protein trafficking and ER-associated protein degradation (ERAD) (Liu and Howell 2010a; Tajima et al. 2008). However, activation of AtbZIP60 is dependent on the ER-localized AtIRE1 proteins in Arabidopsis (Deng et al. 2011; Moreno et al. 2012; Nagashima et al. 2011). Accumulation of misfolded proteins in ER triggers the activation of AtIRE1s, which unconventionally splices AtbZIP60 mRNA in the double stemloops at the cytoplasmic side, causing an open reading frame (ORF) shift and elimination of the transmembrane domain in AtbZIP60 (Deng et al. 2011, 2016). In the nucleus, AtbZIP60 interacts with AtbZIP28 and regulate downstream genes alone or together (Iwata and Koizumi 2005; Song et al. 2015; Tajima et al. 2008). OsNTL3, a homolog of AtNAC062, is up-regulated by OsbZIP74 (ortholog of AtbZIP60) under heat stress conditions and encodes a plasma membrane-tethered membrane associated transcription factor in rice (Hayashi et al. 2012; Liu et al. 2020; Lu et al. 2012; Yang et al. 2014). It is activated and relocates to the nucleus to up-regulate downstream genes involved in UPR and reactive oxygen species (ROS) scavenging, which is essential for heat stress tolerance in rice (Liu et al. 2020).

Relative to vegetative growth, reproductive development is more sensitive to environmental stresses such as heat stress (Barnabas et al. 2008; Begcy et al. 2019; Chaturvedi et al. 2021). In Arabidopsis, the AtbZIP60 promoter is highly active in flowers especially in microspores and tapetum cells, and the bZIP60 mRNA is constitutively spliced/activated in anthers under normal growth conditions suggesting its role in reproductive development (Deng et al. 2016; Iwata et al. 2008). When stressed at reproductive stage, the bzip28 bzip60 double mutant plants were more sensitive to heat stress with short siliques and reduced fertility comparing to that in wild-type plants, which agrees with the important role of these two transcription factors in heat stress responses in reproductive tissues (Zhang et al. 2017).

Previous researches have demonstrated that AtbZIP17, a paralog of AtbZIP28, is activated in a manner similar to AtbZIP28 under salt stress conditions to desensitize the ABA signaling pathway (Liu et al. 2007b, 2008; Zhou et al. 2015). A recent study showed that AtbZIP17 is required for vegetative development together with AtbZIP28 to mediate the expression of multiple genes involved in cell growth (Kim et al. 2018). Although ER stress and heat stress induce the activation of AtbZIP17 (Che et al. 2010), apparently AtbZIP28 and AtbZIP60 are two major transcription factors for canonical UPR gene expression (Kim et al. 2018; Song et al. 2015), the role of AtbZIP17 in heat stress response is not reported. In the current paper, we found that AtbZIP17 also plays important roles in maintaining fertility under heat stress conditions. Through RNA-seq and ChIP-seq analysis, we further revealed that AtbZIP17 directly binds to 113 genes whose expression is dependent on AtbZIP17 in flowers under heat stress conditions.

\section{RESULTS}

\section{AtbZIP17 is essential for maintaining fertility under heat stress conditions}

Our previous transcriptomic data have shown that AtbZIP17 is up-regulated by heat stress in Arabidopsis flower tissues (Zhang et al. 2017). To examine the role of AtbZIP17 in maintaining fertility under heat stress conditions, we compared the heat sensitivity of WT and bzip17 knock-out mutant plants (Liu et al. 2007b) at reproductive stage. Compared to the WT plants, the percentage of type II and type III siliques (reduced fertility) was largely increased in bzip17 mutant plants after exposure to a short period of heat stress $\left(38^{\circ} \mathrm{C}\right)$ at flowering stage (Fig. 1A, B). These results demonstrated that AtbZIP17 is essential for thermotolerance at the reproductive stage in Arabidopsis. 


\section{AtbZIP17 regulates heat stress responsive genes in flowers}

To understand the function of AtbZIP17 in heat stress responses at reproductive stage, we performed RNA-seq analysis of heat-stressed flowers (stage 1-12) in WT and bzip17 mutant plants (Supplemental Fig. S1), and found that 1380 and 493 genes were specifically up-regulated $\left[\log _{2}(\mathrm{FC})>2\right.$ and $\left.P<0.05\right]$ and down-regulated $\left[\log _{2}(\mathrm{FC})<0.5\right.$ and $\left.P<0.05\right]$ in WT flowers, respectively (Fig. 2A). There were 334 and 917 genes that were specifically up-regulated and down-regulated in the flowers of bzip17 mutant plants, respectively (Fig. 2A). Since the activated form of AtbZIP17 has transcriptional activation activity (Liu et al. 2007b), we considered these 1380 genes as AtbZIP17-dependent heat stress responsive genes in flowers (Supplemental Table S1). GO analysis of these genes revealed that 'response to salt stress' and 'response to water deprivation' were enriched (Fig. 2B). At least eight terms related to chloroplast and photosynthesis were also enriched (Fig. 2B), probably because the green tissue sepal was included in the flower samples. These results supported that AtbZIP17 regulates a number of genes involved in stress responses and chloroplast function in Arabidopsis flowers under heat stress conditions.

\section{AtbZIP17 directly binds to the promoters of stress responsive and UPR genes in seedlings}

To reveal genome-wide direct targets of AtbZIP17, we carried out ChIP-seq experiments with heat-stressed MYC-bZIP17 expressing seedling plants. Western blot analysis confirmed that AtbZIP17 is processed in response to heat stress as the small molecular weight band increased under heat stress conditions (Supplemental Fig. S2). The ChIP-seq results showed that MYCbZIP17 was enriched in the $2 \mathrm{~K}$ upstream sequences of totally 1645 genes in 3 replicates (Fig. 3A and Supplemental Table S2). We considered these genes as the AtbZIP17 direct targets in Arabidopsis. For the 133 identified direct targets of AtbZIP28 (Zhang et al. 2017), except AT2G07741, the rest 132 genes are also direct targets of AtbZIP17 as revealed in the current study.

Among the 2629 binding peaks on the promoter regions of these 1645 genes, the bZIP-bind G box and ERSE-II cis-elements were significantly enriched (Fig. 3B), which is similar to the binding characteristics of AtbZIP28 (Liu and Howell 2010b; Zhang et al. 2017). Among the proteins encoded by these target genes, 'response to ABA', 'response to water deprivation', 'endoplasmic reticulum unfolded protein response', and subcellular compartments related to the secretory pathway were all significantly enriched (Fig. 3C). These results suggest that AtbZIP17 has both specific and overlapping direct targets with AtbZIP28.

\section{The expression of AtbZIP17 targets is impaired in flowers of bzip17 mutant plants under heat stress conditions}

To know the direct targets of AtbZIP17 in flowers under heat stress conditions, we compared these bZIP17 direct targets in seedlings with those AtbZIP17-dependent heat stress responsive genes in flowers. The results showed that totally 113 target genes were up-regulated by heat at reproductive stage in WT plants but not in bzip17 mutant plants (Fig. 4A), suggesting that these genes are direct targets of bZIP17 during heat stress response in flowers. GO analysis showed that 'response to salt stress', 'response to water deprivation', 'endoplasmic reticulum UPR', 'ER' and eight terms related to chloroplast and photosynthesis were enriched (Fig. 4B). The dominant role of AtbZIP28/60 in transcriptional control of canonical ER stress gene expression (Song et al. 2015) was confirmed in Arabidopsis seedlings (Supplemental Fig. S3). We also compared these AtbZIP17-dependent heat stress responsive flower genes to those AtbZIP28/60-dependent heat stress responsive flower genes identified in previous study (Zhang et al. 2017), and found that only 59 genes (4.3\%) were also AtbZIP28/60-dependent. Nevertheless, these results revealed direct candidate target genes of AtbZIP17 in flowers and showed that AtbZIP17 has a distinct set of downstream genes to AtbZIP28/60 during heat stress response.

We selected six AtbZIP17 target genes from the ChIPseq analysis, including four canonical UPR genes (CNX1, $S D F 2, S H D$, and $E R D J 3 B$ ) and two salt stress-related genes (ATHB-7 and P5CS1) whose promoter sequences were enriched by AtbZIP17 (Fig. 5A), and conducted ChIP-qPCR. The results indicated that AtbZIP17 indeed binds to the promoter region of all these six genes (Fig. 5B). To check whether the expression of these target genes was affected by AtbZIP17 mutation, RTqPCR was performed. The results showed that all these six genes were significantly up-regulated by heat in WT flowers (Fig. 5C). In contrast, the expression of CNX1, $P 5 C S 1, A T H B-7, E R D J 3 B$ was not significantly affected by heat stress in bzip17 mutant flowers (Fig. 5C). Although the expression of $S D F 2$ and $S H D$ was also significantly up-regulated by heat stress in bzip17 mutant flowers, the fold increase in bzip17 mutant flowers was lower than that in WT flowers (Fig. 5C). Except SDF2, the upregulation of other five genes by heat stress was not much affected in the bzip28 bzip60 double mutant 
plants (Zhang et al. 2017). These results confirmed that AtbZIP17 directly regulates these canonical and noncanonical genes in Arabidopsis flowers.

\section{DISCUSSION}

Plant is sensitive to environmental stresses such as drought, salt and heat stresses at reproductive stage, which often results in yield reduction (Barnabas et al. 2008; Begcy et al. 2019; Liu et al. 2006; Ma et al. 2020). Previous results showed that the silique length is corelated to the number of seeds in Arabidopsis, using this trait, several QTLs associated with thermotolerance at $35{ }^{\circ} \mathrm{C}$ at reproductive stage were identified (BacMolenaar et al. 2015), however, the underlying molecular mechanisms are still largely unknown. Through genome-wide association study (GWAS), a cluster of five Immune-associated nucleotide-binding protein (IAN) genes (IAN2 to IAN6) was identified to be responsible for the variation in heat tolerance at reproductive stage in Arabidopsis (Lu et al. 2021). The IAN proteins were found to promote cell death induced by heat stress, ER stress, and cell death-inducing molecules (Lu et al. 2021). The association of ER stress response with male reproductive development is widely recognized (Deng et al. 2016; Fragkostefanakis et al. 2016) and several mutants defective in proteins related to protein quality control in plants have pollen development phenotype at normal growth temperature (Singh et al. 2021; Sun et al. 2021). The ER stress response is also essential for thermotolerance at reproductive stage (Singh et al. 2021), and knock-out mutations of AtbZIP28 and AtbZIP60, two major UPR regulators in Arabidopsis, confer heat sensitivity at reproductive stage (Zhang et al. 2017). In the current paper, we showed that AtbZIP17 is also important for thermotolerance at reproductive stage, these findings advance our understanding on maintaining fertility under heat stress conditions in plants.

Although AtbZIP17 is activated following treatment with ER stress inducing reagent (Che et al. 2010), the function of AtbZIP17 in canonical ER stress is still an open question. The up-regulation of canonical ER stress genes such as $B i P 1 / 2 / 3$ and $C R T 1$ by ER stress was completely blocked in bzip28 bzip60 double mutant plants (Song et al. 2015; Sun et al. 2013), and the truncated form of AtbZIP17 did not while the truncated form of AtbZIP28 did activate the promoter of BiP3 in the transient expression assay in protoplasts (Tajima et al. 2008). These results support that AtbZIP17 is not involved in canonical UPR. However, AtbZIP17 has overlapping direct targets with AtbZIP28 as identified in

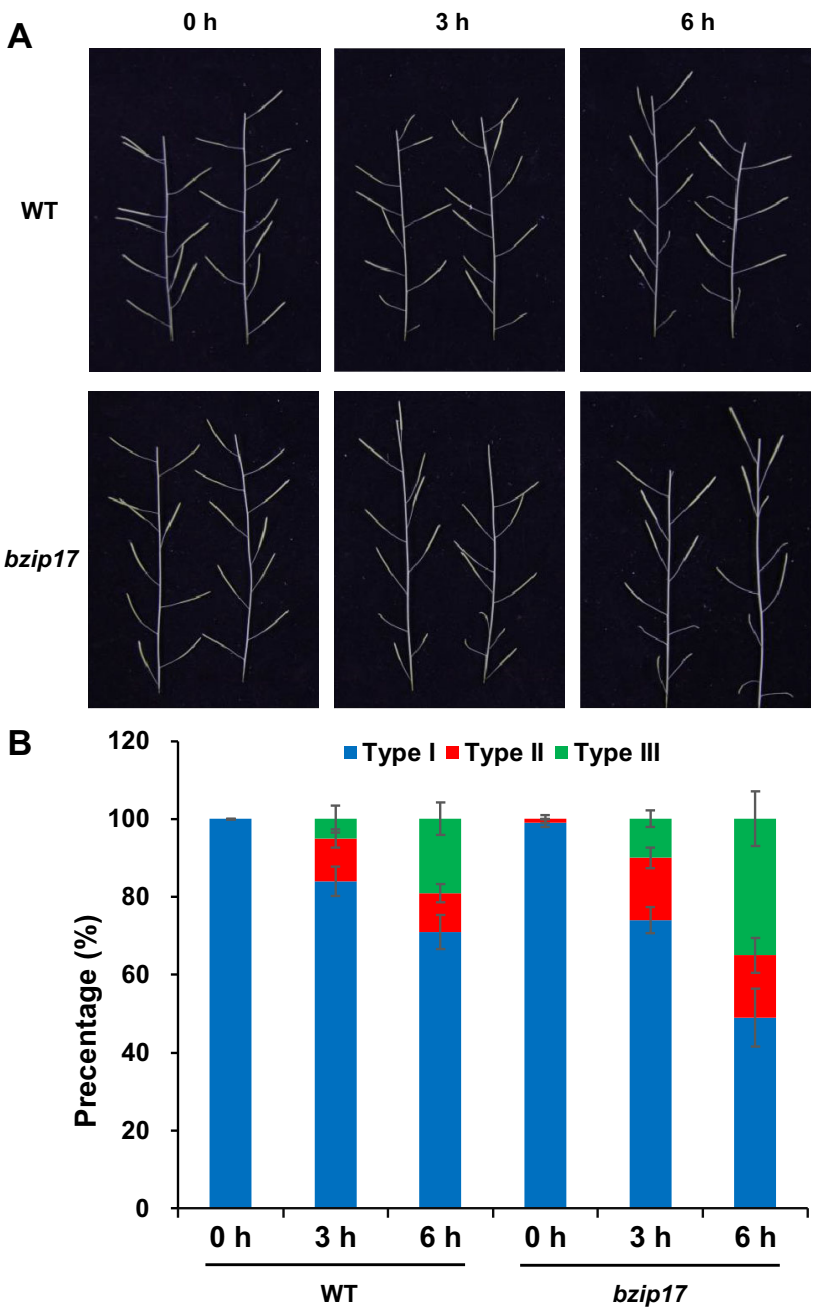

Fig. 1 AtbZIP17 is required for maintaining fertility under heat stress conditions. A Siliques of Arabidopsis wild-type plants (WT) and AtbZIP17 mutant (bzip17) plants at recovery stage after treating with heat stress for different time at the stage of late bolting. B Heat stress sensitivity of WT and bzip17 plants in terms of silique length. Silique lengths were measured and percentage of each type of silique was calculated. Type I, full fertile $(>10 \mathrm{~mm}$ in length); Type II, partial sterile (5-10 $\mathrm{mm}$ in length); Type III, complete sterile $(<5 \mathrm{~mm}$ in length). Bars depict SE $(n=10)$

the current study, many of which are known to be important for UPR in plants. Thus, it is possible that AtbZIP17 is still involved in canonical UPR in seedlings, but that function is dependent on the function of AtbZIP28/60, which awaits further investigation in future. The expression of AtbZIP17/28 is not while that of AtbZIP60 is up-regulated by canonical ER stress in Arabidopsis seedlings (Tajima et al. 2008), while the expression of all the three genes AtbZIP17/28/60 is upregulated by heat stress in Arabidopsis flowers (Zhang et al. 2017), suggesting that AtbZIP17 is functional redundant to AtbZIP28/60. This is well agreed to our results in the current study that 'endoplasmic reticulum 
Fig. 2 AtbZIP17 regulates downstream genes in flowers under heat stress conditions. A Venn diagrams showing the common and specific heat responsiveness in flowers of wild-type plants (WT) and AtbZIP17 mutant (bzip17) plants. B GO analysis of AtbZIP17-dependent heat responsive genes in flowers

\section{A}
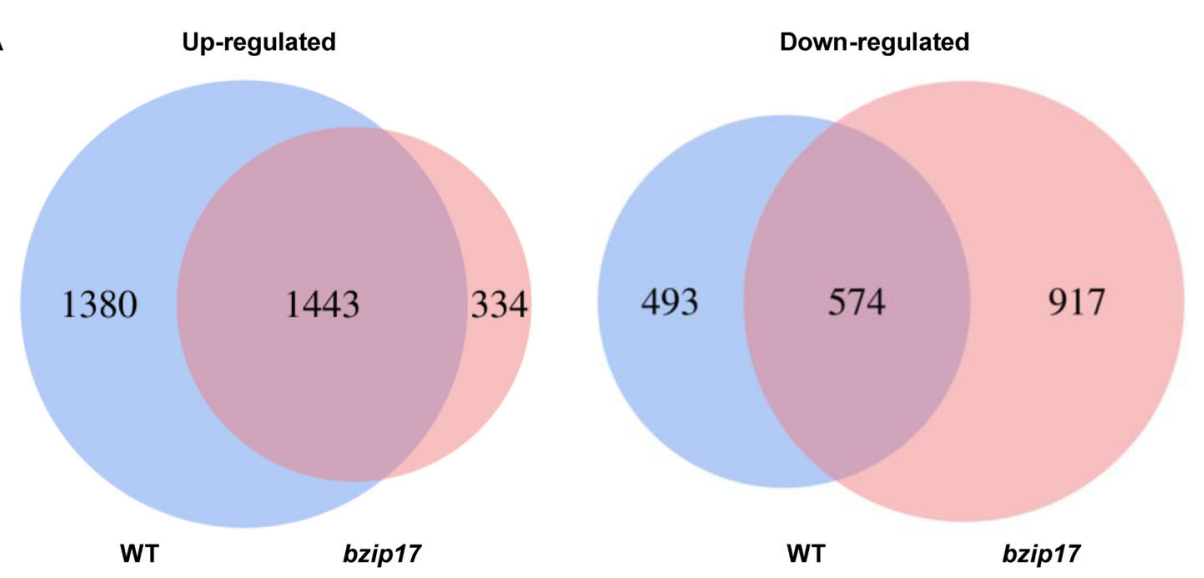

B
UPR' is enriched in the AtbZIP17 direct targets in flowers during heat stress responses. These findings are important for understanding the role of UPR in heat stress response at reproductive stage in plants.

AtbZIP17 regulates vegetative development at normal growth temperature conditions by regulating a different branch of downstream genes to AtbZIP28/60 (Bao et al. 2019; Kim et al. 2018). Several genes such as $A T H B-7, L T P 3$, and $P P 2 C$-like were previously identified as the downstream genes of AtbZIP17 under salt stress condition (Henriquez-Valencia et al. 2015; Liu et al. 2007b, 2008). Excessive salt treatment may affect the function and/or constituent of ER, therefore, triggering ER stress response and activation of the ER-associated transcription factor AtbZIP17. However, such ER stress response could be non-canonical UPR (Howell 2017). Indeed, AtbZIP17 is activated by AtS2P following ABA treatment through regulating $A T H B-7$ to desensitize the
ABA signaling (Zhou et al. 2015). ATHB-7 is a direct target of AtbZIP17, and it is up-regulated by heat stress in flowers which is dependent on AtbZIP17, therefore, AtbZIP17 is involved in ABA signaling during heat stress responses in reproductive tissues in plants.

The GO terms related to chloroplast were enriched in the AtbZIP17 direct targets in flowers during heat stress responses, probably because green sepals were included in the flower samples. Chloroplasts, the photosynthetic organelles of plants, are highly sensitive to heat stress (Hu et al. 2020; Sun et al. 2020). On the other hand, chloroplasts are major resources for ROS production and play an important role in retrograde signaling to protect the integrity and function of chloroplast (Kim 2020; Sun and Guo 2016). Our current results suggest that AtbZIP17 is also important for chloroplast development/function during heat stress response in plants. 
Fig. 3 AtbZIP17 directly binds to the promoter of target genes in heat-stressed seedlings. A Venn diagram showing the direct targets of AtbZIP17 in three replicates. B AtbZIP17-binding motifs in the binding peaks of AtbZIP17 targets. C GO analysis of AtbZIP17 direct targets
A

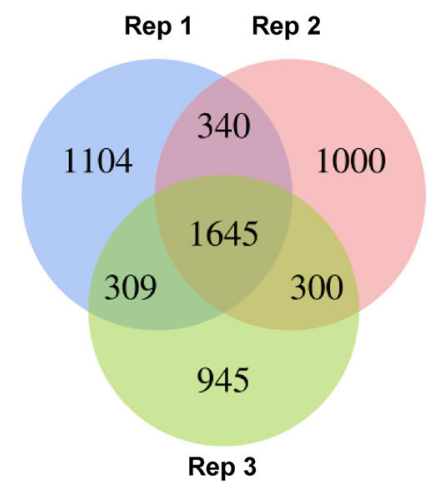

C

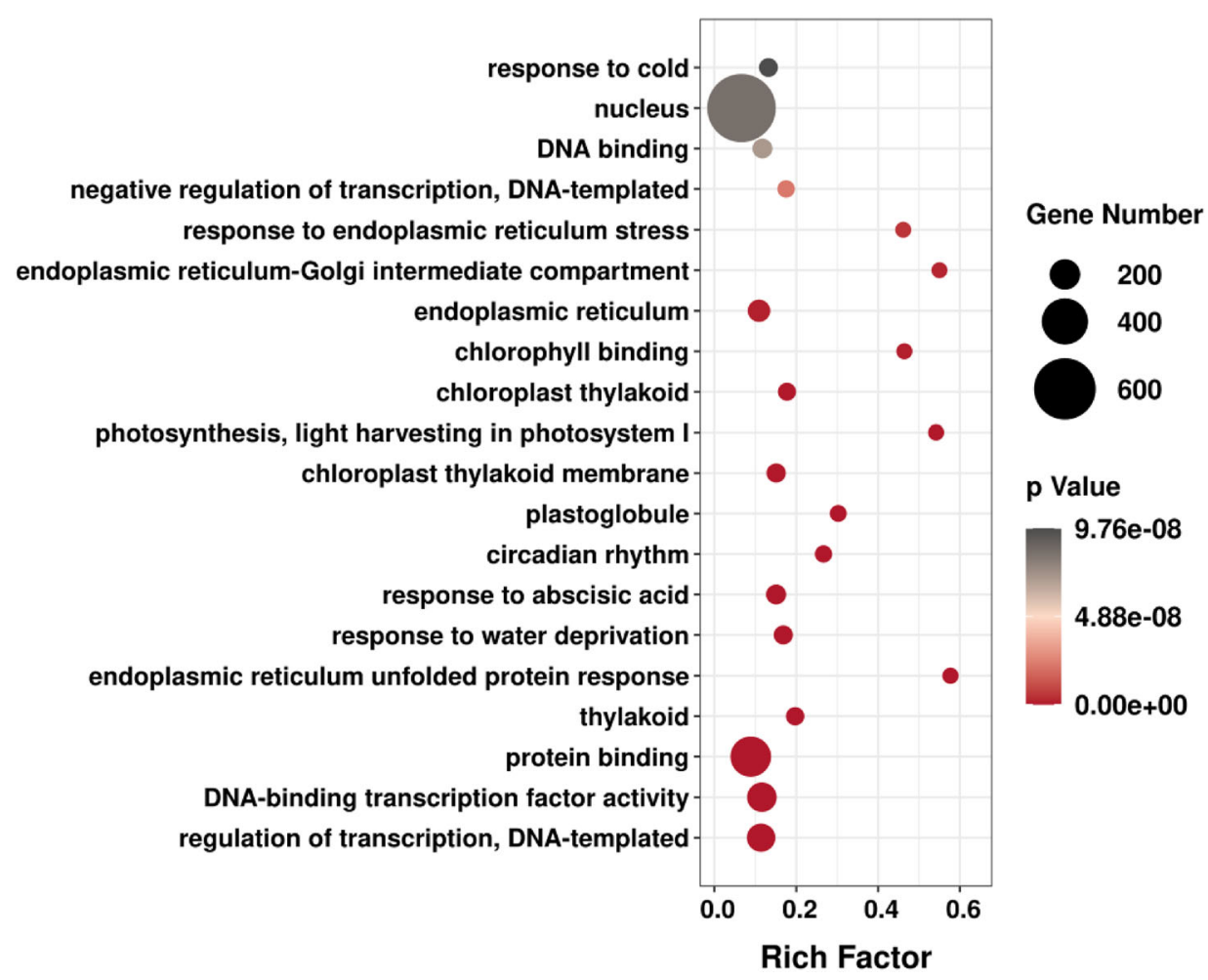

B
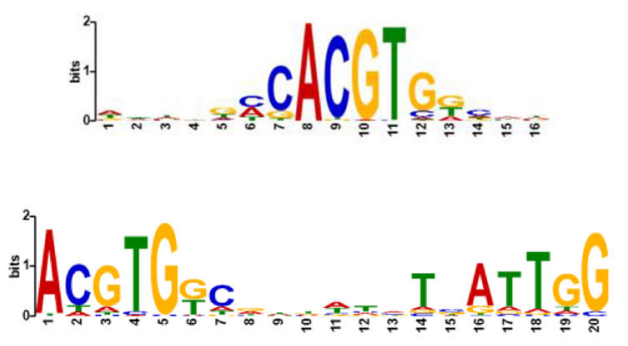

\section{MATERIALS AND METHODS}

\section{Plant material and growing conditions}

All plants in this study are in Columbia-0 (Col-0) background. The T-DNA insertional mutant bzip17 (SALK_104326) was obtained from the Arabidopsis Biological Resource Center (ABRC) (Liu et al. 2007b). The seeds were washed with $0.01 \%$ sodium hypochlorite solution for $20 \mathrm{~min}$, and then washed five times with sterile water in an ultra-clean bench. They were grown directly on half-strength Murashige and Skoog (MS) medium (with $1.2 \%$ sucrose and $0.7 \%$ agar, $\mathrm{pH}$ 5.7). After 3 days of vernalization at $4{ }^{\circ} \mathrm{C}$, they were transferred to a standard plant incubator $\left(22^{\circ} \mathrm{C}, 16 / 8-\mathrm{h}\right.$ day/night and $60 \%$ humidity). For phenotypic analysis and RNA-seq analysis, the seedlings were grown in an incubator for 10 days and then transplanted into a commercial soilless (mixed which include peat, vermiculite, and perlite) under the same conditions.

\section{Phenotype analysis}

Phenotypic analysis of heat stress sensitivity during reproductive stage was performed as followings. After grown at $22{ }^{\circ} \mathrm{C}$ until flowering, the unopened floral buds (approximately stage 12) were marked with colored threads/marks, and then placed in the incubator at $38{ }^{\circ} \mathrm{C}$ for $3 \mathrm{~h}$ and $6 \mathrm{~h}$, respectively. The control plants were grown at $22{ }^{\circ} \mathrm{C}$ with the same condition. After heat 
Fig. 4 The expression of AtbZIP17 targets is affected in bzip17 mutant flowers under heat stress conditions. A Venn diagram showing 113 direct candidate targets of AtbZIP17 in flowers under heat stress conditions. B GO analysis of heat responsive AtbZIP17 direct targets in flowers. C Comparison of AtbZIP17dependent heat responsive genes in flowers and AtbZIP28/60-dependent heat responsive genes in flowers
A

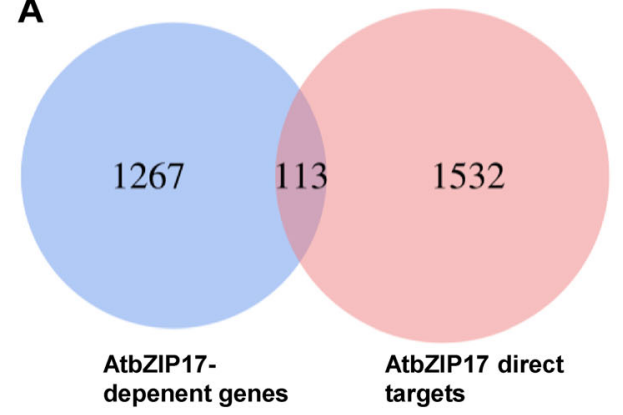

C

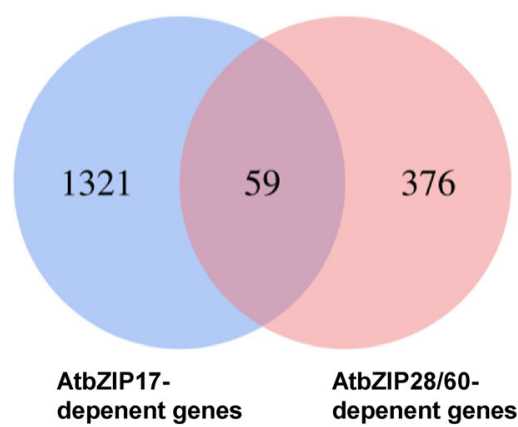

B

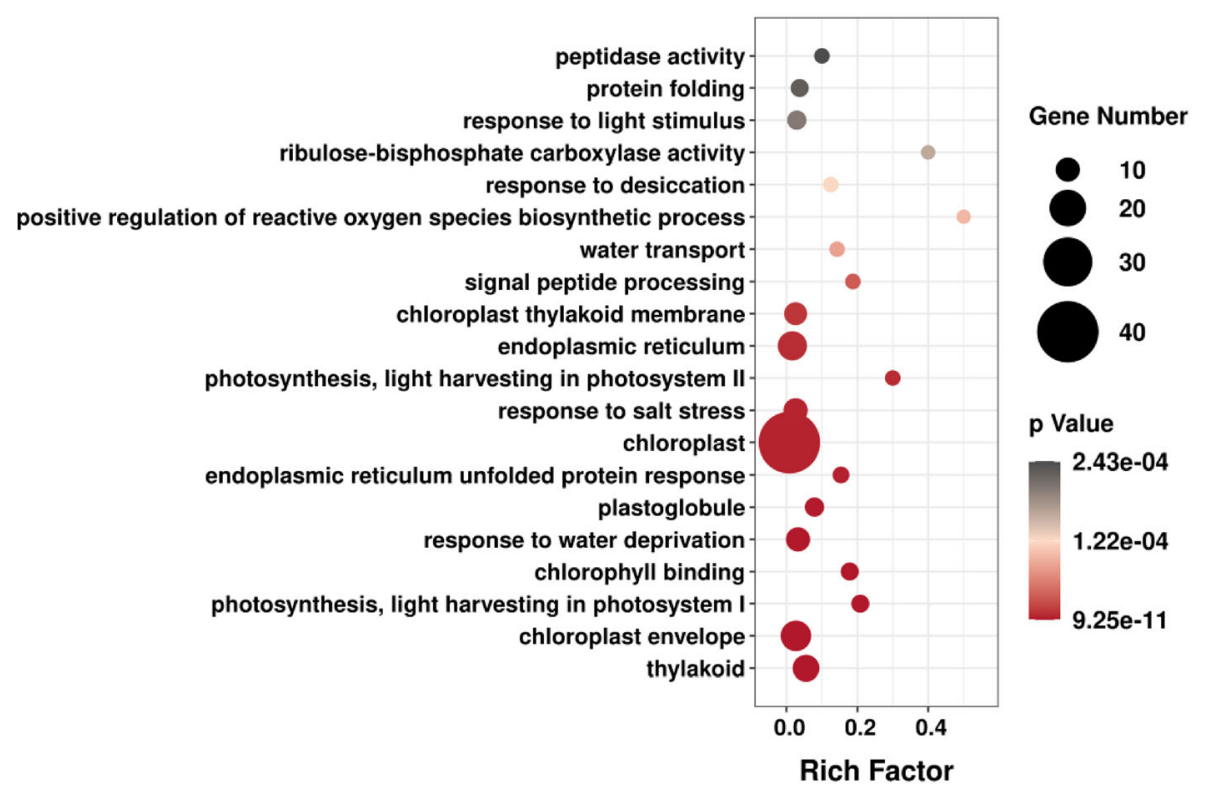

stress treatment, the plants were transferred to $22{ }^{\circ} \mathrm{C}$ and grew further for 10 days. The main inflorescence starting from the mark was selected, and the length of 10 siliques from the mark was measured in ten biological replicates. The siliques were divided into three categories: fully fertile (type I, length $>10 \mathrm{~mm}$ ), partially sterile (type II, length $5-10 \mathrm{~mm}$ ) and completely sterile (type III, length $<5 \mathrm{~mm}$ ) according to the protocols in our previous study (Zhang et al. 2017).

\section{RNA-seq and RT-qPCR analysis}

For RNA-seq analysis, wild-type and bzip17 mutant plants at flowering stage were treated at $38{ }^{\circ} \mathrm{C}$ for $6 \mathrm{~h}$, and the control plants were kept at $22^{\circ} \mathrm{C}$. The unopened floral buds (stage 1-12) from eight plants were collected and immediately frozen in liquid nitrogen with a total of three biological replicates for each treatment. Total RNA was extracted with Trizol (Invitrogen, Shanghai, USA). The cDNA library was constructed according to standard protocols on Illumina Novaseq ${ }^{\mathrm{TM}}$ 6000 (LC-Bio Technology Co., Ltd., Hangzhou, China). Cutadapt software is used to remove reads that contain adapter contamination, and to remove low-quality bases and undetermined bases. The HISAT2 software was used to map the reads to the genome and assembled by StringTie with default parameters. Then, all transcriptomes from all samples were merged to reconstruct a comprehensive transcriptome using gffcompare software. After the final transcriptome was generated, StringTie and ballgown were used to estimate the expression levels of all transcripts by calculating FPKM (fragments per kilo-base of transcript per million of mapped reads). The differentially expressed mRNAs were selected with fold change $>2$ or fold change $<0.5$ and $P$ value $<0.05$ by $\mathrm{R}$ package edgeR or DESeq 2 , and then gene ontology (GO) enrichment analysis was conducted. For RT-qPCR, total RNAs were extracted with RNA Prep Pure Plant kits (Tiangen, Beijing, China) and reverse transcribed using $5 \times$ PrimeScript RT Master 

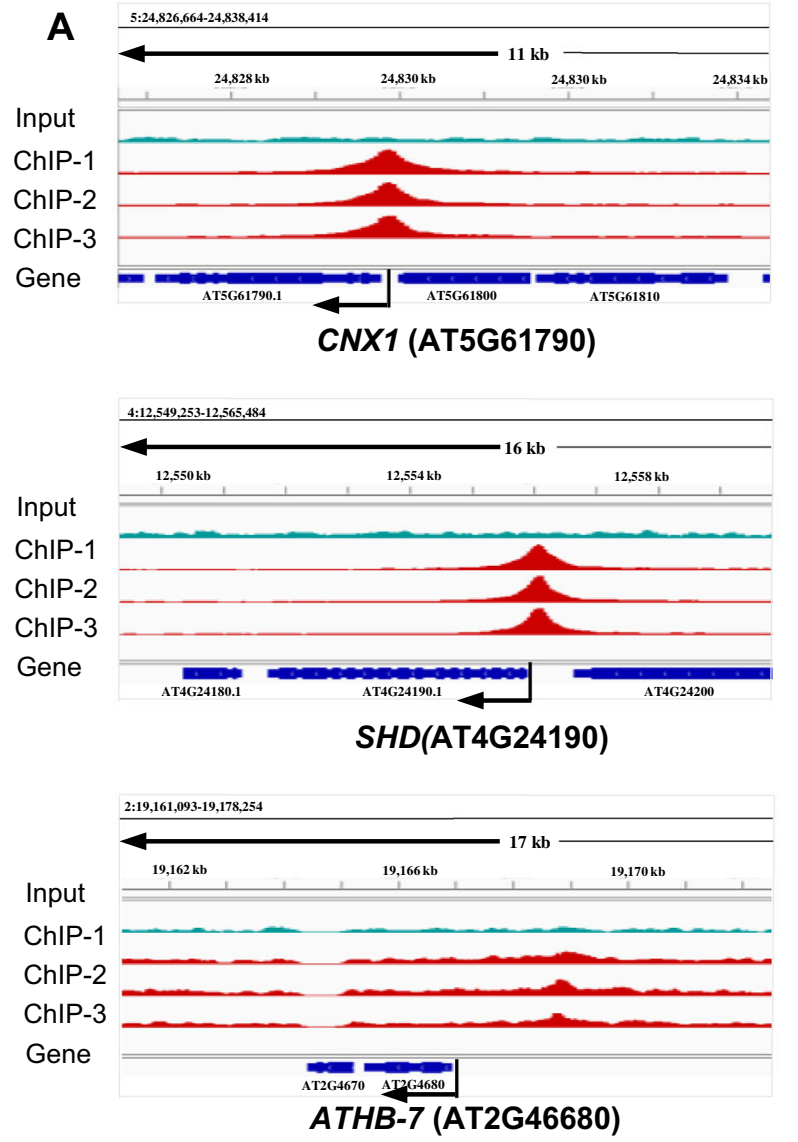

B

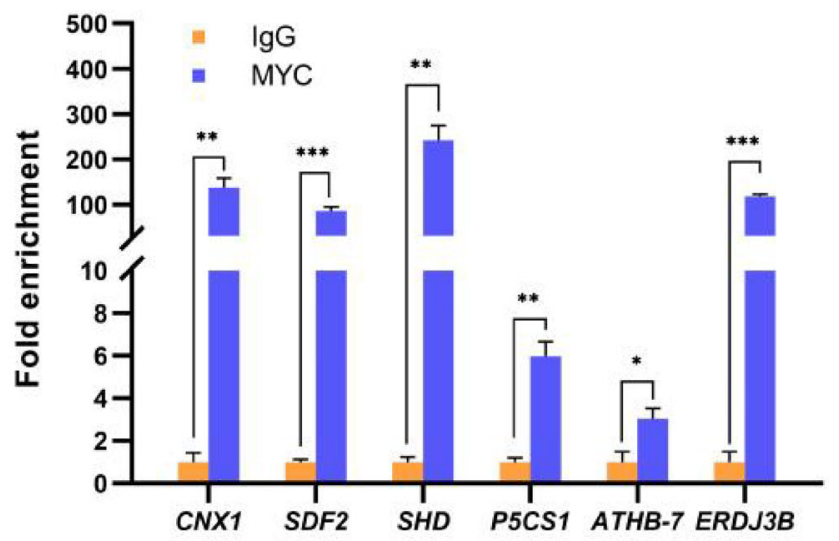

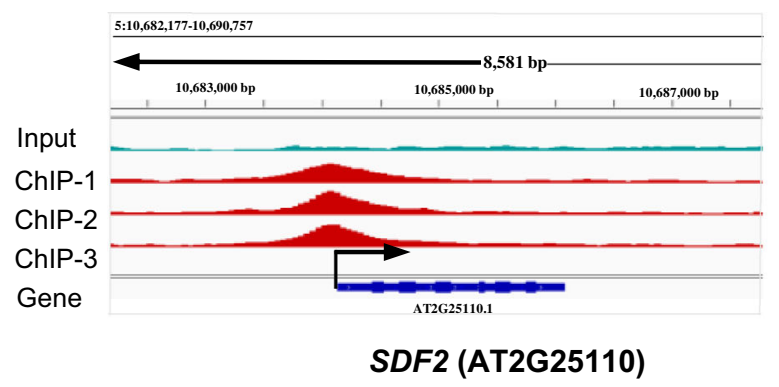
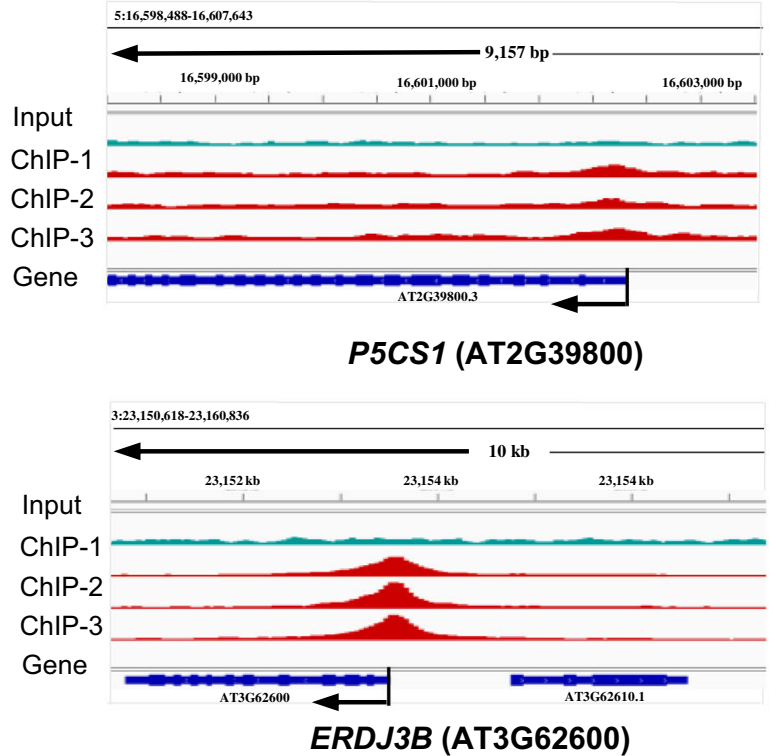

C

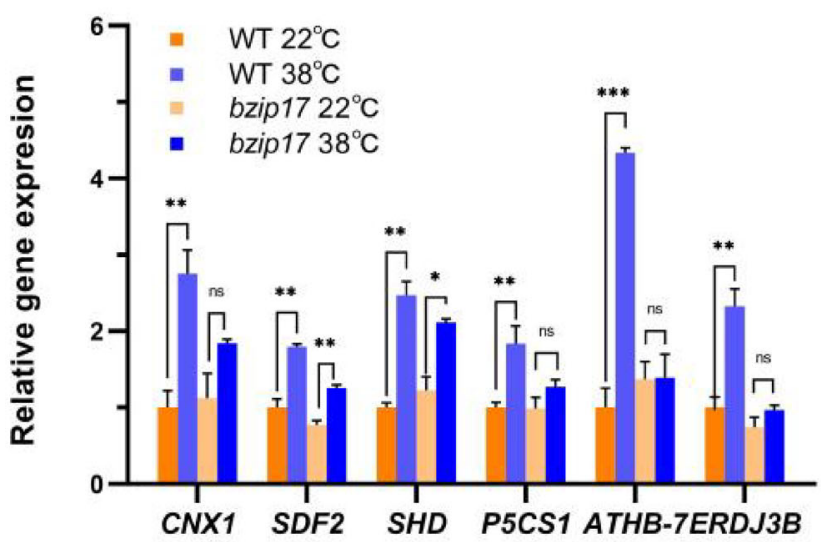

Fig. 5 Mutation of AtbZIP17 impairs the up-regulation of AtbZIP17 direct targets in flowers under heat stress conditions. A Distribution of AtbZIP17 binding peaks on six representative target genes in the Integrated Genome Browser. Aligned reads are indicated in red (heattreated, three replicates) and cyan (input). B ChIP-qPCR analysis. Transgenic seedlings overexpressing MYC-bZIP17 were heat-stressed and harvested for ChIP-qPCR using anti-MYC antibody. IgG was used as a negative control. Relative enrichment of each sample was normalized to that of IgG sample, both of which were normalized to that of the TA3 control. C RT-qPCR analysis of AtbZIP17 target genes in flowers under heat stress conditions. Relative gene expression is the expression level of each gene normalized to that in the WT plants, both of which were normalized to that of $P P 2 A$. The bars depict the $S E(n=3)$. ${ }^{* * *}(P<0.001) ;{ }^{* *}(P<0.01)$; ${ }^{*}(P<0.05)$; ns, (not significant at $P<0.05$ ) in $t$ tests

Mix (Takara, Dalian, China). RT-qPCR was performed with SuperReal PreMix Color kits (Tiangen, Beijing, China) in a CFX96 real-time system (Bio-Rad, USA).
Gene expression level was calculated based on the $\Delta \Delta \mathrm{Ct}$ (threshold cycle) method. All the primers are included in Supplemental Table S3. 


\section{ChIP-seq assay}

For Chromatin immuno-precipitation coupled with highthroughput sequencing (ChIP-seq), 13-day-old $35 \mathrm{~S}_{\text {pro:- }}$ :MYC-bZIP17 seedlings grown at $22{ }^{\circ} \mathrm{C}$ were placed at $42{ }^{\circ} \mathrm{C}$ for $2 \mathrm{~h}$, and the seedlings were immediately placed in a $1 \%(\mathrm{v} / \mathrm{v})$ formaldehyde solution $[0.4 \mathrm{M}$ sucrose, 10 mM Tris-HCl (pH 8.0), 1 mM EDTA (pH 8.0) $1 \%$ formaldehyde and PMSF] under vacuum for fixing the protein and DNA, and $0.125 \mathrm{M}$ glycine was added to terminate the reaction. Samples were washed with sterile water and grinded into powder in liquid nitrogen for ChIP-seq according to previous protocols (Zhang et al. 2017). Briefly, the powder was mixed with the lysis buffer [50 mM HEPES (pH 7.5), $150 \mathrm{mM} \mathrm{NaCl}, 1 \mathrm{mM}$ EDTA, 1\% Triton X-100, 5 mM $\beta$-mercaptoethanol, 10\% Glycerol and proteinase inhibitor cocktail (Roche, USA)], and then sonicated to obtain almost $200-500$ bp chromatin fragment. Protein A-agarose beads (Millipore) and an anti-MYC antibody (Sigma-Aldrich, USA) were used to precipitate the chromatin. The ChIP-DNA and INPUT DNA libraries were constructed and sequenced by Genergy Bio (Shanghai, China) with Illumina Novaseq $^{\mathrm{TM}} 6000$. Skewer software was used to remove lowquality and linker sequence fragments, and to filter sequences less than $50 \mathrm{bp}$. Bowtie was used to perform unique mapping analysis between the filtered sequencing data and the reference genome to further remove duplicated reads. MACS software was used to do the peak calling. In each replicate, compared with the input, there were higher binding peak intensity and $P$ values $<0.001$, which was considered as the potential binding sites of AtbZIP17. The related genes where the common binding peak region in the upstream $2 \mathrm{~K}$ in three replicates were considered as the potential targets of AtbZIP17. The common binding cis-elements were identified using MEME and GO analysis was performed using the R package edgeR. For ChIP-qPCR, 13-day-old seedlings of $35 \mathrm{~S}_{\text {pro }}:: \mathrm{MYC}$-bZIP17 plants were treated or nontreated with high temperature at $42{ }^{\circ} \mathrm{C}$ for $2 \mathrm{~h}$ and sampled for ChIP-qPCR. Primers were listed in Supplemental Table S3.

\section{Western blot analysis}

To verify heat-induced activation of MYC-bZIP17, 13-day-old seedlings of $35 \mathrm{~S}_{\text {pro: }}:$ MYC-bZIP17 plants grown at $22{ }^{\circ} \mathrm{C}$ were placed in an incubator at $42{ }^{\circ} \mathrm{C}$ for $2 \mathrm{~h}$, and the plants at $22^{\circ} \mathrm{C}$ were used as the controls. Samples were extracted with SDS protein extraction buffer [125 mM Tris-HCl (pH 8.0), $375 \mathrm{mM} \mathrm{NaCl,}$ $2.5 \mathrm{mM}$ EDTA, 1\% SDS and 1\% $\beta$-mercaptoethanol] and separated on $4-20 \%(\mathrm{w} / \mathrm{v})$ SDS-PAGE gels. The proteins were transferred with nitrocellulose membranes and MYC-bZIP17 was detected using anti-MYC antibody (Sigma-Aldrich, USA) or anti-Tubulin (Sigma-Aldrich, USA) with Tanon-5200 Chemiluminescence Imaging System (Tanon, Shanghai, China).

\section{Accession numbers}

RNA-Seq and ChIP-Seq data from this article can be found in Gene Expression Omnibus (GEO) under the accession number (GSE184984).

Supplementary InformationThe online version contains supplementary material available at https://doi.org/10.1007/ s42994-021-00062-1.

Acknowledgements This project was financially supported by grants from the National Natural Science Foundation of China (Grant Nos. 31872653 and 31800210), the Zhejiang Provincial Talent Program (Grant No. 2019R52005), and the Fundamental Research Funds for the Zhejiang Provincial Universities (Grant No. 2021XZZX023).

Author contributions JXL conceived the project, JG, MJW, JJW, and HPL performed the experiments, JG and JXL designed the experiments, analysed the data, and wrote the paper.

\section{Declarations}

Conflict of interest On behalf of all authors, the corresponding author states that there is no conflict of interest. Author Jian-Xiang Liu was not involved in the journal's review of this manuscript.

Open Access This article is licensed under a Creative Commons Attribution 4.0 International License, which permits use, sharing, adaptation, distribution and reproduction in any medium or format, as long as you give appropriate credit to the original author(s) and the source, provide a link to the Creative Commons licence, and indicate if changes were made. The images or other third party material in this article are included in the article's Creative Commons licence, unless indicated otherwise in a credit line to the material. If material is not included in the article's Creative Commons licence and your intended use is not permitted by statutory regulation or exceeds the permitted use, you will need to obtain permission directly from the copyright holder. To view a copy of this licence, visit http://creativecommons.org/ licenses/by/4.0/.

\section{References}

Bac-Molenaar JA, Fradin EF, Becker FFM, Rienstra JA, van der Schoot J, Vreugdenhil D, Keurentjes JJB (2015) Genome-wide association mapping of fertility reduction upon heat stress reveals developmental stage-specific QTLs in Arabidopsis thaliana. Plant Cell 27:1857-1874

Bao Y, Bassham DC, Howell SH (2019) A functional unfolded protein response is required for normal vegetative development. Plant Physiol 179:1834-1843 
Barnabas B, Jaeger K, Feher A (2008) The effect of drought and heat stress on reproductive processes in cereals. Plant Cell Environ 31:11-38

Begcy K, Nosenko T, Zhou LZ, Fragner L, Weckwerth W, Dresselhaus $T$ (2019) Male sterility in maize after transient heat stress during the tetrad stage of pollen development. Plant Physiol 181:683-700

Chaturvedi P, Wiese AJ, Ghatak A, Zaveska Drabkova L, Weckwerth W, Honys D (2021) Heat stress response mechanisms in pollen development. New Phytol 231:571-585

Che P, Bussell JD, Zhou WX, Estavillo GM, Pogson BJ, Smith SM (2010) Signaling from the endoplasmic reticulum activates brassinosteroid signaling and promotes acclimation to stress in Arabidopsis. Sci Signal 3:141

Deng Y, Humbert S, Liu JX, Srivastava R, Rothstein SJ, Howell SH (2011) Heat induces the splicing by IRE1 of a mRNA encoding a transcription factor involved in the unfolded protein response in Arabidopsis. Proc Natl Acad Sci USA 108:7247-7252

Deng Y, Srivastava R, Quilichini TD, Dong H, Bao Y, Horner HT, Howell SH (2016) IRE1, a component of the unfolded protein response signaling pathway, protects pollen development in Arabidopsis from heat stress. Plant J 88:193-204

Ding Y, Shi Y, Yang S (2020) Molecular regulation of plant responses to environmental temperatures. Mol Plant 13:544-564

Fragkostefanakis S, Mesihovic A, Hu Y, Schleiff E (2016) Unfolded protein response in pollen development and heat stress tolerance. Plant Reproduction 29:81-91

Gao H, Brandizzi F, Benning C, Larkin RM (2008) A membranetethered transcription factor defines a branch of the heat stress response in Arabidopsis thaliana. Proc Natl Acad Sci USA 105:16398-16403

Guo M, Liu JH, Ma X, Luo DX, Gong ZH, Lu MH (2016) The plant heat stress transcription factors (HSFs): structure, regulation, and function in response to abiotic stresses. Front Plant Sci $7: 114$

Hayashi S, Wakasa Y, Takahashi H, Kawakatsu T, Takaiwa F (2012) Signal transduction by IRE1-mediated splicing of bZIP50 and other stress sensors in the endoplasmic reticulum stress response of rice. Plant J 69:946-956

Henriquez-Valencia C, Moreno AA, Sandoval-Ibanez O, Mitina I, Blanco-Herrera F, Cifuentes-Esquivel N, Orellana A (2015) bZIP17 and bZIP60 regulate the expression of BiP3 and other salt stress responsive genes in an UPR-independent manner in Arabidopsis thaliana. J Cell Biochem 116:1638-1645

Howell SH (2017) When is the unfolded protein response not the unfolded protein response? Plant Sci 260:139-143

Hu SS, Ding YF, Zhu C (2020) Sensitivity and responses of chloroplasts to heat stress in plants. Front Plant Sci 11:375

Iwata Y, Koizumi N (2005) An Arabidopsis transcription factor, AtbZIP60, regulates the endoplasmic reticulum stress response in a manner unique to plants. Proc Natl Acad Sci USA 102:5280-5285

Iwata Y, Fedoroff NV, Koizumi N (2008) Arabidopsis bZIP60 is a proteolysis-activated transcription factor involved in the endoplasmic reticulum stress response. Plant Cell 20:3107-3121

Jacob P, Hirt H, Bendahmane A (2017) The heat-shock protein/ chaperone network and multiple stress resistance. Plant Biotechnol J 15:405-414

Kim C (2020) ROS-driven oxidative modification: its impact on chloroplasts-nucleus communication. Front Plant Sci 10:1729

Kim JS, Yamaguchi-Shinozaki K, Shinozaki K (2018) ER-anchored transcription factors bZIP17 and bZIP28 regulate root elongation. Plant Physiol 176:2221-2230
Lesk C, Rowhani P, Ramankutty N (2016) Influence of extreme weather disasters on global crop production. Nature 529:84-87

Li XM, Chao DY, Wu Y, Huang XH, Chen K, Cui LG, Su L, Ye WW, Chen H, Chen HC, Dong NQ, Guo T, Shi M, Feng Q Zhang P, Han B, Shan JX, Gao JP, Lin HX (2015) Natural alleles of a proteasome alpha 2 subunit gene contribute to thermotolerance and adaptation of African rice. Nat Genet 47:827-833

Liu JX, Howell SH (2010a) bZIP28 and NF-Y transcription factors are activated by ER Stress and assemble into a transcriptional complex to regulate stress response genes in Arabidopsis. Plant Cell 22:782-796

Liu JX, Howell SH (2010b) Endoplasmic reticulum protein quality control and its relationship to environmental stress responses in plants. Plant Cell 22:2930-2942

Liu JX, Howell SH (2016) Managing the protein folding demands in the endoplasmic reticulum of plants. New Phytol 211:418-428

Liu JX, Liao DQ, Oane R, Estenor L, Yang XE, Li ZC, Bennett J (2006) Genetic variation in the sensitivity of anther dehiscence to drought stress in rice. Field Crop Res 97:87-100

Liu JX, Srivastava R, Che P, Howell SH (2007a) An endoplasmic reticulum stress response in Arabidopsis is mediated by proteolytic processing and nuclear relocation of a membraneassociated transcription factor, bZIP28. Plant Cell 19:4111-4119

Liu JX, Srivastava R, Che P, Howell SH (2007b) Salt stress responses in Arabidopsis utilize a signal transduction pathway related to endoplasmic reticulum stress signaling. Plant J 51:897-909

Liu JX, Srivastava R, Howell SH (2008) Stress-induced expression of an activated form of AtbZIP17 provides protection from salt stress in Arabidopsis. Plant Cell Environ 31:1735-1743

Liu XH, Lyu YS, Yang W, Yang ZT, Lu SJ, Liu JX (2020) A membraneassociated NAC transcription factor OsNTL3 is involved in thermotolerance in rice. Plant Biotechnol J 18:1317-1329

Lu SJ, Yang ZT, Sun L, Sun L, Song ZT, Liu JX (2012) Conservation of IRE1-regulated bZIP74 mRNA unconventional splicing in rice (Oryza sativa L.) involved in ER stress responses. Mol Plant 5:504-514

Lu S, Zhu TQ, Wang ZX, Luo LL, Wang S, Lu MH, Cui YM, Zou BH, Hua J (2021) Arabidopsis immune-associated nucleotidebinding genes repress heat tolerance at the reproductive stage by inhibiting the unfolded protein response and promoting cell death. Mol Plant 14:267-284

Ma XW, Su Z, Ma H (2020) Molecular genetic analyses of abiotic stress responses during plant reproductive development. J Exp Bot 71:2870-2885

Moreno AA, Mukhtar MS, Blanco F, Boatwright JL, Moreno I, Jordan MR, Chen Y, Brandizzi F, Dong X, Orellana A, PajerowskaMukhtar KM (2012) IRE1/bZIP60-mediated unfolded protein response plays distinct roles in plant immunity and abiotic stress responses. PLoS ONE 7:e31944

Nagashima Y, Mishiba KI, Suzuki E, Shimada Y, Iwata Y, Koizumi N (2011) Arabidopsis IRE1 catalyses unconventional splicing of bZIP60 mRNA to produce the active transcription factor. Sci Rep. https://doi.org/10.1038/srep00029

Nover L, Scharf KD (1997) Heat stress proteins and transcription factors. Cell Mol Life Sci 53:80-103

Singh MB, Lohani N, Bhalla PL (2021) The role of endoplasmic reticulum stress response in pollen development and heat stress tolerance. Front Plant Sci 12:661062

Song ZT, Sun L, Lu SJ, Tian Y, Ding Y, Liu JX (2015) Transcription factor interaction with COMPASS-like complex regulates histone H3K4 trimethylation for specific gene expression in plants. Proc Natl Acad Sci USA 112:2900-2905 
Sun AZ, Guo FQ (2016) Chloroplast retrograde regulation of heat stress responses in plants. Front Plant Sci 7:398

Sun L, Lu SJ, Zhang SS, Zhou SF, Sun L, Liu JX (2013) The lumenfacing domain is important for the biological function and organelle-to-organelle movement of bZIP28 during ER Stress in Arabidopsis. Mol Plant 6:1605-1615

Sun J, Tian Y, Lian Q Liu JX (2020) Mutation of DELAYED GREENING1 impairs chloroplast RNA editing at elevated ambient temperature in Arabidopsis. J Genet Genomics 47:201-212

Sun JL, Li JY, Wang MJ, Song ZT, Liu JX (2021) Protein quality control in plant organelles: current progress and future perspectives. Mol Plant 14:95-114

Tajima H, Iwata Y, Iwano M, Takayama S, Koizumi N (2008) Identification of an Arabidopsis transmembrane bZIP transcription factor involved in the endoplasmic reticulum stress response. Biochem Biophys Res Commun 374:242-247
Yang ZT, Lu SJ, Wang MJ, Bi DL, Sun L, Zhou SF, Song ZT, Liu JX (2014) A plasma membrane-tethered transcription factor, NAC062/ANAC062/NTL6, mediates the unfolded protein response in Arabidopsis. Plant J 79:1033-1043

Zhang SS, Yang H, Ding L, Song ZT, Ma H, Chang F, Liu JX (2017) Tissue-specific transcriptomics reveals an important role of the unfolded protein response in maintaining fertility upon heat stress in Arabidopsis. Plant Cell 29:1007-1023

Zhang JY, Li XM, Lin HX, Chong K (2019) Crop improvement through temperature resilience. Annu Rev Plant Biol 70:753-780

Zhou SF, Sun L, Valdés AE, Engström P, Song ZT, Lu SJ, Liu JX (2015) Membrane-associated transcription factor peptidase, site-2 protease, antagonizes ABA signaling in Arabidopsis. New Phytol 208:188-197 